\title{
Retrospective Study of 133 Canadian Hidradenitis Suppurativa Patients and Literature Review
}

\author{
Erika L. Crowley ${ }^{1}$, Claudia J. Posso-De Los Rios ${ }^{2}$, Melinda J. Gooderham ${ }^{1,3,4 *}$ \\ ${ }^{1}$ SKiN Centre for Dermatology, Peterborough, ON, \\ ${ }^{2}$ Dermatology Program, University of Toronto, Toronto, ON \\ ${ }^{3}$ Queen's University, Kingston, ON, \\ ${ }^{4}$ Probity Medical Research, Waterloo, ON
}

${ }^{\star}$ Correspondence to: Melinda J. Gooderham, Queen’s University, Kingston, ON, 775 Monaghan Road South, Peterborough, ON K9J 5K2; E-mail: mgooderham@ centrefordermatology.com

Received: February 22, 2018; Accepted: March 12, 2018; Published: March 16, 2018;

\begin{abstract}
Introduction: Hidradenitis Suppurativa (HS) is a chronic inflammatory condition involving a recalcitrant course with a significant impact on quality of life. Biologics are gaining popularity as a treatment for HS management. Intralesional corticosteroid injections have been useful in treating localized inflammatory dermatoses including HS.

Methods and results: The medical charts of a community dermatology practice were reviewed for patients diagnosed with HS from January 1 , 2006 to July 1, 2015. Information collected included the patients' demographics, clinical characteristics, comorbidities, and treatments prior to and post dermatologic consultation. A total of 133 patients were identified. A female to male ratio of $3.75: 1$, mean age of onset of $27.0+12.6$ years, and mean delay of diagnosis of $6.7+8.0$ years was determined. Of these patients, $42.9 \%$ were Hurley stage I, $49.6 \%$ were Hurley stage II, and $7.5 \%$ were Hurley stage III. Tobacco exposure accounted for $49.6 \%$ of patients. The most common HS treatments included topical antibiotics $(66.9 \%)$ and systemic antibiotics $(54.9 \%)$, followed by intralesional steroids (36.8\%), spironolactone (18.0\%), oral isotretinoin (7.5\%), oral contraceptives (6.8\%), and biologics $(6.0 \%)$.

Conclusions: The HS prevalence and treatment application in a dermatologic practice were closely related to existing literature. Biologics are a suitable alternative for patients with moderate-to-severe HS, alone or with comorbidities. Intralesional steroids are an effective option in the treatment of localized disease in acute flares, either as monotherapy or adjuvant therapy due to their anti-inflammatory effect. Controlled randomized clinical studies are required to achieve more definitive results.
\end{abstract}

Keywords: Hidradenitis suppurativa, Hurley Stage, comorbidities, delay in diagnosis, adalimumab, intralesional corticosteroid injections

\section{Introduction}

Hidradenitis Suppurativa (HS) is a chronic, recurrent, inflammatory disease characterized by lesions affecting apocrine gland bearing skin. Patients with HS often present with comedones, inflammatory and non-inflammatory nodules, and abscesses, which can result in sinus tract formation and scarring [1]. The Hurley's classification describes disease severity; stage I involves the abscess formation; stage II involves tract and scar tissue formation, with recurrent abscesses and single or multiple separated lesions; and stage III involves interconnected tracts and abscesses throughout an entire anatomical locations [1,2]. HS pathogenesis remains unclear. One theory describes HS as an immune mediated disease caused by an abnormal pilosebaceous-apocrine unit, followed by follicular occlusion, perifollicular cyst formation, and finally indigenous microorganism trapping that ruptures into the dermis [3,4]. Genetics and lifestyle are also thought to play a role in HS development and management [2-4].

Although relatively unknown among the general public, HS has an estimated prevalence ranging from $0.05-4 \%$ of the population $[2,5]$.
Numerous studies have demonstrated that HS has a negative impact on quality of life and sexual health when compared to other chronic skin conditions [6-8]. The location, severity, and pain associated with the disease makes patients uncomfortable and embarrassed. Despite the debilitating nature of HS, there is currently no cure and the treatment goal is to control symptoms and inflammatory manifestations. Management plans typically involve both drug intervention and lifestyle changes. In this literature and retrospective chart review, the real-world prevalence of HS characteristics and application of treatments in a dermatologic practice were described, highlighting the early use of biologics and intralesional corticosteroid injections.

\section{Methods}

There were 133 medical charts identified from a community dermatology practice in Ontario, Canada during the period of January 1,2006 to July 1, 2015. Patients diagnosed with HS that had undergone at least one dermatologic assessment were included in the study. Information collected included patient demographics, age at onset and diagnosis, type and location of lesions, Hurley Stage, treatments prior to and following dermatologic consultation, current status, and follow-up length. 


\section{Results}

\section{Patient characteristics}

Clinical characteristics of the 133-patient population are shown in Table 1. The mean age of HS onset was $27.0+12.6$ years (range 9-62 years) and the mean delay in diagnosis was $6.7+8.0$ years (maximum 30 years). There were 3.75 times more females than males, as 105 (78.9\%) patients were female and $28(21.1 \%)$ were male. A family history of HS was specified by only $8(6.0 \%)$ patients. Tobacco use, including current $53(39.8 \%)$ and former 13 (9.8\%), was declared by 66 (49.6\%) patients. Several comorbidities were also reported by some of our patients, as 38 (28.6\%) had obesity, 15 (11.3\%) diabetes mellitus, 7 (5.3\%) inflammatory bowel disease (IBD), 6 (4.5\%) psoriasis, 3 (2.3\%) polycystic ovary syndrome (PCOS), and 3 (2.3\%) spondyloarthritis.

Table 1: Characteristics and comorbidities of HS patient population.

\begin{tabular}{|c|c|c|}
\hline Clinical Characteristics & n & $\%$ \\
\hline Total Patients & 133 & \\
\hline Age of onset, mean $+\mathrm{SD}, \mathrm{y}$ & $27.0 \pm 12.6$ & \\
\hline Delay of diagnosis, mean $+\mathrm{SD}, \mathrm{y}$ & $6.7 \pm 8.0$ & \\
\hline Males & 28 & 21.1 \\
\hline Females & 105 & 78.9 \\
\hline Family history & 8 & 6.0 \\
\hline Tobacco exposure (Previous or current) & 66 & 49.6 \\
\hline \multicolumn{3}{|l|}{ Comorbidities } \\
\hline Obesity & 38 & 28.6 \\
\hline Diabetes & 15 & 11.3 \\
\hline IBD & 7 & 5.3 \\
\hline Psoriasis & 6 & 4.5 \\
\hline PCOS & 3 & 2.3 \\
\hline Spondyloarthritis & 3 & 2.3 \\
\hline \multicolumn{3}{|l|}{ Hurley stages } \\
\hline Hurley stage I & 57 & 42.9 \\
\hline Hurley stage II & 66 & 49.6 \\
\hline Hurley stage III & 10 & 7.5 \\
\hline \multicolumn{3}{|l|}{ Anatomical location } \\
\hline Inguinal & 88 & 66.2 \\
\hline Axilla & 81 & 60.9 \\
\hline Lower abdomen and pubis region & 34 & 25.6 \\
\hline Inframammary and intermammary & 29 & 21.8 \\
\hline Thighs & 24 & 18 \\
\hline Anogenital & 19 & 14.3 \\
\hline Buttocks & 17 & 12.8 \\
\hline Neck and scalp & 4 & 3.0 \\
\hline \multicolumn{3}{|l|}{ IBD = Inflammatory Bowel Disease } \\
\hline PCOS = Polycystic Ovary Syndrome & & \\
\hline
\end{tabular}

Hurley stage I and II were predominant, accounting for 57 (42.9\%) and $66(49.6 \%)$ patients, respectively. Hurley Stage III was present in $10(7.5 \%)$ patients. In terms of anatomic location, $88(66.2 \%)$ patients had HS in the inguinal area, $81(60.9 \%)$ axilla, 34 (25.6\%) lower abdomen and pubic region, $29(21.8 \%)$ inframammary and intermammary region, 24 (18\%) thighs, 19 (14.3\%) anogenital region, 17 (12.8\%) buttocks, and 4 (3.0\%) neck and scalp.

\section{Treatments prior to and following dermatologic consultation}

Patients used various therapies prior to and following dermatologic consultation, as shown in Table 2. Prior to dermatologic consultation, systematic antibiotics were the most common form of HS treatment accounting for $63(47.4 \%)$ patients, followed by topical antibiotics for $33(24.8 \%)$ patients, incision and drainage (I\&D) for 24 (18.0\%), surgery referral for 15 (11.3\%), oral contraceptives (OCP) for 11 (8.3\%), spironolactone for $5(3.8 \%)$, oral isotretinoin for $5(3.8 \%)$, intralesional corticosteroid (ILC) injections for $3(2.3 \%)$, biologics for $1(0.8 \%)$, and laser for $1(0.8 \%)$. There was no finasteride treatment before dermatologic consultation.

Table 2: Treatments used for HS patients prior to and following dermatologist consultation.

\begin{tabular}{|l|c|c|}
\hline \multirow{2}{*}{ Treatment } & Pre-Consultation, & $\begin{array}{c}\text { After } \\
\text { Consultation, }\end{array}$ \\
\cline { 2 - 3 } & $\mathbf{n}$ & $\mathbf{n}$ \\
\hline Topical antibiotics & $33(24.8 \%)$ & $89(66.9 \%)$ \\
\hline Systemic Antibiotics & $63(47.4 \%)$ & $73(54.9 \%)$ \\
\hline ILC & $3(2.3 \%)$ & $49(36.8 \%)$ \\
\hline Spironolactone (Female only) & $5(3.8 \%)$ & $24(18.0 \%)$ \\
\hline Oral isotretinoin & $5(3.8 \%)$ & $10(7.5 \%)$ \\
\hline OCP (Female only) & $11(8.3 \%)$ & $9(6.8 \%)$ \\
\hline Biologics & $1(0.8 \%)$ & $8(6.0 \%)$ \\
\hline Surgery referral & $15(11.3 \%)$ & $2(1.5 \%)$ \\
\hline Finasteride & $1(0.8 \%)$ & $1(0.8 \%)$ \\
\hline Laser & $24(18.0 \%)$ & 0 \\
\hline I\&D & $0.0 \%)$ \\
\hline $\begin{array}{l}\text { ILC = Intralesional Corticosteroid injections } \\
\text { I\&D = Incision and Drainage }\end{array}$ & & \\
\hline
\end{tabular}

There was a shift in prescribed medications following dermatologic consultation. Topical antibiotics were prescribed to 89 (66.9\%) patients and systemic antibiotics to $73(54.9 \%)$ patients. ILC injections were administered to $49(36.8 \%)$ patients. In addition, spironolactone use increased to $24(18.0 \%)$ patients after dermatologic consultation, oral isotretinoin to $10(7.5 \%)$, biologics to $8(6.0 \%)$, finasteride to 1 $(0.8 \%)$, and laser therapy use remained at $1(0.8 \%)$ patient. OCP was used by $9(6.8 \%)$ patients, I\&D was not performed after dermatologic consultation, and 8 (6.0\%) patients were referred for surgery. 


\section{Biologics}

Of the 8 patients who received biologics, $5(62.5 \%)$ patients used adalimumab, 5 (62.5\%) used infliximab, and 2 (25.0\%) used etanercept, as shown in Table 3. The mean months of follow up was $24.2+27.9$ months (ongoing maximum of 73 months). Six (75.0\%) patients continue ongoing biologic treatment. In addition, 6 (75\%) patients receiving biologics also received ILC injections. Etanercept, prescribed for psoriasis in some patients, reduced the psoriasis symptoms but had no response for HS. Of the 5 patients who received infliximab, 4 showed HS improvement. Overall, 1 stopped infliximab due to pruritus, 1 stopped due to urticarial lesions and dyspnea, and 1 switched biologics to better manage their psoriasis and reported worsened HS while off infliximab. Of the 5 patients who received adalimumab, 4 showed HS improvement and 1 stopped due to a selflimited leg edema.

Table 3: Patients with biologic or intralesional corticosteroid injection therapy.

\begin{tabular}{|c|c|c|}
\hline Therapy & $\mathbf{n}$ & $\%$ \\
\hline \multicolumn{3}{|l|}{ Biologics } \\
\hline Total Patients & 8 & \\
\hline Adalimumab & 5 & 62.5 \\
\hline Infliximab & 5 & 62.5 \\
\hline Etanercept & 2 & 25 \\
\hline Follow up in months, mean, $+\mathrm{SD}$ & $24.2+27.9$ & \\
\hline Treatment ongoing & 6 & 75 \\
\hline \multicolumn{3}{|c|}{ Intralesional Corticosteroid Injections (ILC) } \\
\hline Total patients & 49 & \\
\hline Total Injections & 165 & \\
\hline \multicolumn{3}{|l|}{ Concentration of ILC injections } \\
\hline $2.5 \mathrm{mg} / \mathrm{ml}$ & 8 & 12.1 \\
\hline $3.3 \mathrm{mg} / \mathrm{ml}$ & 23 & 34.8 \\
\hline $5 \mathrm{mg} / \mathrm{ml}$ & 29 & 43.9 \\
\hline $10 \mathrm{mg} / \mathrm{ml}$ & 6 & 9.1 \\
\hline \multicolumn{3}{|l|}{ Visits for ILC injections } \\
\hline$<2$ & 32 & 65.3 \\
\hline $3-4$ & 9 & 18.4 \\
\hline$>5$ & 8 & 16.3 \\
\hline Follow up in months, mean, $\pm \mathrm{SD}$ & $10.3 \pm 17.6$ & \\
\hline
\end{tabular}

\section{Intralesional corticosteroid injections}

Forty-nine (36.8\%) patients received ILC and a total of 165 injections were administered, as shown in Table 3. The most common ILC concentration was $5 \mathrm{mg} / \mathrm{mL}$, which was received by 29 (43.9\%) patients, followed by $3.3 \mathrm{mg} / \mathrm{ml}$ by 23 (34.8\%), $2.5 \mathrm{mg} / \mathrm{ml}$ by $8(12.1 \%)$, and $10 \mathrm{mg} / \mathrm{ml}$ by $6(9.1 \%)$ patients. There were $25(51 \%)$ patients that received ILC injections at a single visit, $16(32.7 \%)$ at 2 to 4 visits, and $8(16.3 \%)$ patients at 5 or more visits. The mean months of follow up after the first ILC injection was 10.3 months +17.6 months (ongoing maximum of 73 months).

\section{Discussion}

\section{Patient characteristics}

Age of onset and delay in diagnosis: Although HS has been reported in children, the disease typically develops after puberty [2]. Consistent with recent literature, the mean age of onset in this study was $27.0+12.6$ years (range $9-62$ years). Others have reported an average onset age between 20 and 24 years, with an estimation that $2.0 \%$ of HS patients develop the disease before 11 years of age $[9,10]$. Early-onset HS patients have been found to have a significantly higher family history of the disease [10].

The diagnostic delay associated with HS is a well-known problem [11]. The mean delay of diagnosis in this study of $6.7+8.0$ years (maximum of 30 years) is consistent with reports of typical HS diagnostic delays ranging from 5 to 14 years [12]. Similarly, a study with 517 HS patients from 24 different countries found a global mean delay in diagnosis of $7.2+8.7$ years (maximum of 41 years) [11].

Female prevalence: HS is known for having a higher prevalence in females than males [2]. There was a 3.75:1 female-to-male ratio in this study. Ratios are typically reported as approximately 3.1, with some variance $[2,13,14]$. Ratios have been recorded from as low as $2: 1$ to as high as 5:1 in patient populations [15]. Determining prevalence can be challenging because there is a delay in diagnosis and not all HS patients seek treatment.

Family history, tobacco use and comorbidities: HS is believed to have a genetic component. Although only 8 (6.0\%) of our patients self-reported a family history of HS, the nature of this retrospective study and awareness of the disease may have affected this frequency. HS family history was not routinely captured in clinic encounters. A study using questionnaires specifically inquiring about the family history of HS patients found that $38 \%$ of the participants had at least one family member affected by HS [16]. Other studies have found that approximately one-third of their patient population had a HS family history, supporting the claim that family histories are associated with earlier disease onset, longer duration, and more extensive disease $[13,17]$. In addition, there has been evidence linking autosomal dominance to some HS cases $[18,19]$. HS has been linked to chromosome 1p21.1-1q25.3 and $\gamma$-secretase complex mutations in some cases where the HS patients had concurrent severe acne and perifolliculitis capitis abscedens et suffodiens (dissecting cellulitis of the scalp) [20].

Although the connection between tobacco smoking and HS is not completely understood, smoking is thought to trigger disease onset and increase disease severity [2]. Similar to current literature, 66 (49.6\%) patients in this study were self-proclaimed current or exsmokers. Other studies have found higher rates of tobacco smoking patients in the range of $66-71 \%$ current and $8-15 \%$ ex-smokers $[17,21$ 23]. Despite the large frequency of HS patients that continue to smoke, studies have shown that smoking cessation contributed to increased incidences of self-reported HS remission [24,25]. Patients should be reminded of the association between smoking and exacerbation of the disease. 
In our study, we identified obesity, diabetes mellitus, IBD, psoriasis, PCOS, and spondyloarthritis as comorbidities in some of our patients, which has been also reported in a large retrospective study of 2292 patients [26]. HS typically associates with many concomitant and secondary diseases, contributing to disease severity [26-28].

Hurley stages and anatomical locations: The Hurley stage severity and anatomical locations of the HS lesions in this patient population were consistent with current literature for this disease [13,17,18,22,29]. The inguinal and axilla regions are the most commonly reported HS locations. Our results were similar to a large Dutch population, where $45.5 \%$ of patients had Hurley stage I, $41.5 \%$ had Hurley stage II and $13.0 \%$ had Hurley stage III [18]. Other studies have reported populations from 24.1-68.2\% Hurley stage I, 27.6-53.7\% Hurley stage II and 2.2\%-22.2\% Hurley stage III [13,22,29].

\section{Treatment}

HS treatment can be difficult because management must be individualized per the disease location and severity. When developing a treatment plan, the dermatologist assessed availability and accessibility of appropriate medications for each patient. Medical treatments aim to decrease bacterial load, reduce follicular occlusion, decrease immune responses, alter hormonal balance, improve wound healing, reduce pain, and improve patient quality of life. Due to the chronic and uncontrollable nature of the disease, HS treatment goals aim to provide stability and comfort. The dermatologist initiated several treatments not previously provided by primary care, such as spironolactone, isotretinoin, ILC, and biologics. Other treatments prescribed by both primary and specialist care included topical and systemic antibiotics, OCP, surgery referrals, finasteride, I\&D, and laser therapy.

Topical and systemic antibiotics: Topical and systemic antibiotics were the most commonly prescribed therapy in this study. Antibiotics, used for their anti-inflammatory effect, are often a firstline therapy for HS $[2,30]$. Systemic antibiotics, in short courses, were the most commonly prescribed pre-consultation treatment. Topical antibiotics were most commonly prescribed following dermatologic consultation, which is not surprising given that topical clindamycin is a recommended evidence-based first-line therapy [30].

Hormonal therapy: Spironolactone, finasteride, and oral contraceptives: With anti-androgen activity, spironolactone, finasteride, and OCP are typically used for female patients if they are not responding to conventional therapy or have a history of hormonal imbalance. Spironolactone was a common prescription after dermatologic consultation. Finasteride was not used prior to dermatologic consultation but it was used after consultation. OCP was used more often by primary care prior to dermatologic consultation, however; it is not captured if this was prescribed for the purposes of controlling HS or for birth control.

Although research with anti-androgens is limited, spironolactone has been used successfully in several case series [31-33]. In particular, 17 of 20 patients in a recent case series found spironolactone to be effective [32]. Spironolactone has potential as first-line therapy for women with mild-to-moderate HS [32].
Finasteride is not a first-choice treatment due to the side effect profile. To date, finasteride use has been documented for both male and female HS patients, including adolescents [34]. Outcomes with finasteride have been overall favourable as concomitant therapy with antibiotics or surgery $[2,34]$.

Oral isotretinoin: Oral isotretinoin use increased after dermatologic consultation. This option was favoured when patients had concomitant acne also requiring treatment. Literature results with oral isotretinoin, however, have been mixed. A literature review of 174 patients from 7 studies concluded that there was significant improvement in $18 \%$, moderate improvement in $17 \%$ and no response in $64 \%$ of the patients included [35]. Patients that benefited from the immunomodulatory effects of isotretinoin had mild HS [35].

Surgical approaches: Surgical referrals were less common in this patient population after dermatologic consultation because a medical approach was preferred for HS. Typical surgical procedures for severe HS, including deroofing, skin-tissue-saving excision with electrosurgical peeling, and wide excision, can lead to relatively high patient satisfaction [36,37]. Surgical success is limited by accessibility to surgeons who are willing to operate on this population, who are often smokers that may have wound healing issues.

Although I\&D was the third most common modality preconsultation, it was not a preferred treatment approach at this dermatology clinic. I\&D is typically used in the emergency room as a method to alleviate acute symptoms. While I\&D provides temporary relief by decompressing and emptying nodules, the lesion typically reoccurs overtime [38].

Laser therapy: Laser therapy was used by few patients in this study. Laser and light-based therapies aim to reduce HS flares by decreasing the number of hair follicles, sebaceous glands, and bacteria in HS-affected regions, as well as by debulking chronic problematic lesions [39]. This therapy is also limited by accessibility in many regions, including ours.

Biologic agents: Biologics have been successful in treating inflammatory diseases for many years. Several literature reviews advocate biologic use for HS, though formal placebo controlled studies are limited [40,41]. Eight (6.0\%) patients in this study used biologics for HS therapy. Since this chart review was completed soon after adalimumab was approved for HS, most biologic use was off-label at the time. This resulted in difficulty securing reimbursement coverage by most insurance companies and was therefore prescribed mainly for associated comorbidities (psoriasis, IBD). Since then, adalimumab has been approved by Health Canada, Food and Drug Administration, and the European Medicines Agency for the treatment of adults with moderate-to-severe HS and is used more commonly in our clinic but not captured in this chart review.

Although biologics were prescribed mainly to treat comorbidities, they were successful in treating HS inflammatory symptoms. The mean time of follow up, $24.2+27.9$ months (ongoing maximum of 73 months), for the patients using biologics, showed continuity of treatment over time. At the 2015 cutoff, 6 (75\%) of 8 patients had ongoing biologic treatment. Many of the patients, 6 (75\%), who were 
on a biologic agent were also receiving ILC injections as an adjunctive therapy.

There is a lack of good evidence supporting the use of etanercept for HS management and the 2 cases in this study were prescribed etanercept for psoriasis. While these patients showed good response to etanercept for psoriasis, there was no HS response. Although an initial etanercept open-label study showed promising results [42], following studies have not been able to reproduce the results $[43,44]$. Currently, etanercept is not recognized as a suitable biologic for HS management $[2,40,41]$. Adalimumab and infliximab have been more thoroughly studied with promising results.

While taking infliximab for psoriasis, 4 (80\%) reported HS improvement, however; some patients in this series stopped taking infliximab due to adverse events of pruritus, urticarial lesions, and dyspnea. The HS symptoms did worsen once the patients discontinued infliximab. One single-center randomized trial $(\mathrm{N}=38)$ showed improvement over 8 weeks in subjects receiving infliximab over placebo in the HS severity index. Although the primary end-point did not reach statistical significance, there was clinical and statistical significance of multiple secondary end-points [45]. Although our sample size was small, recent studies have shown that while both infliximab and adalimumab are effective at treating moderate-tosevere HS, adalimumab may be more tolerable $[46,47]$.

Adalimumab was well tolerated and effective in our patient population, as 4 (80\%) patients reported good responses and continued on treatment. Only 1 patient stopped treatment due to a self-limited leg edema. The best evidence supporting adalimumab use for HS comes from the PIONEER I and PIONEER II trials, which showed a significantly greater clinically meaningful improvement with weekly adalimumab (40 mg) compared to placebo [48]. Since the time of this review, additional biologics beyond the anti-TNF class are being explored for HS treatment including those targeting IL-1beta (anakinra) [49], IL-17 (secukinumab) [50,51], and IL-12/23 (ustekinumab) [52,53].

Intralesional corticosteroid injections: Although not a common form of treatment pre-dermatologic-consultation, ILC was the third most used form of therapy in our series, accounting for 49 (36.8\%) patients. Injections were typically administered as concomitant therapy in localized inflammatory lesions. ILC injections are practical, simple, cost-effective, and readily available in dermatology clinics. Many reviews have advocated their use [2,40,54-56]. ILC injections, utilized as both monotherapy and adjunctive therapy, work effectively for localized lesions and acute flares. Specifically, ILC can cause rapid reduction of single recalcitrant inflammatory nodules typically within days $[2,57]$. Complications, like superinfection, are rare and side-effects with the recommended treatment dosage are uncommon $[54,55]$.

Earlier studies have demonstrated intralesional triamcinolone as an effective treatment for acne cysts [58,59]. ILC was used in one study to control existing lesions in one of four HS patients [31]. A case series made the observation that all 11 of their patients with concurrent HS and pyoderma gangrenosum had at least partial success with intralesional triamcinolone injections during the course of their treatment [60]. A more recent case series assessed the outcomes using ILC in $36 \mathrm{HS}$ patients and found that the injections were successful in reducing pain after 1 day and inflammation after 7 days [57]. Despite this, the amount of clinical data based on the use of ILC for HS is currently lacking. Controlled studies would further support using ILC for HS.

\section{Conclusions}

This retrospective study determined the HS characteristics and treatments in a real-world Canadian dermatology practice. Epidemiology findings, similar to previous literature reports, were established. Topical and systemic antibiotics were common HS treatments in this study population. The dermatologist initiated several treatments such as ILC, spironolactone, biologics, and isotretinoin. Biologics are a feasible alternative for patients with moderate-to-severe HS, alone or with comorbidities. ILC is a suitable and easily available option for acute HS therapy. Biologics as primary therapy, and ILC as adjuvant therapy, achieved positive responses for HS management in the moderate-to-severe patient population. Additional studies are required to evaluate the impact of biologics and intralesional injections for HS management in controlled clinical trials.

\section{References}

1. Hurley HJ (1989) “Axillary Hyperhidrosis, Apocrine Bromhidrosis, Hidradenitis Suppurativa, and Familial Benign Pemphigus: Surgical approach” In: Roenigk RK, Roenigk HH (eds), Dermatologic Surgery, New York: Dekker pp. 729-739.

2. Deckers IE, Prens EP (2016) An Update on Medical Treatment Options for Hidradenitis Suppurativa. Drugs 76: 215-229. [Crossref]

3. Prens E, Deckers I (2015) Pathophysiology of hidradenitis suppurativa: an update. $J$ Am Acad Dermatol 73(5)S8-11.

4. Hoffman LK (2017) Pathophysiology of hidradenitis suppurativa. Semin Cutan Med Surg 36(2): 47-54.

5. Lachaine $J$ (2016) The prevalence and incidence of hidradenitis suppurativa in canada: results from a population-based survey. Value Health 19(3): 123.

6. Alavi A (2015) Quality-of-life impairment in patients with hidradenitis suppurativa: a Canadian study. Am J Clin Dermatol 16(1): 61-65.

7. Kouris A (2016) Quality of life and psychosocial implications in patients with hidradenitis suppurativa. Dermatol 232(6): 687-691.

8. Janse IC (2017) Sexual health and quality of life are impaired in hidradenitis suppurativa: a multicentre cross-sectional study. Br J Dermatol (176)1042-1047.

9. Deckers IE, van der Zee HH, Boer J, Prens EP (2015) Correlation of early-onset hidradenitis suppurativa with stronger genetic susceptibility and more widespread involvement. J Am Acad Dermatol 72: 485-488. [Crossref]

10. Palmer RA, Keefe M (2001) Early-onset hidradenitis suppurativa. Clin Exp Dermatol 26: 501-503. [Crossref]

11. Saunte DM (2015) Diagnostic delay in hidradenitis suppurativa is a global problem. Br J Dermatol 173(6): 1546-1549.

12. Jemec GB, Kimball AB (2015) Hidradenitis suppurativa: Epidemiology and scope of the problem. J Am Acad Dermatol 73(5): S4-S7.

13. Canoui-Poitrine F (2009) Characteristics of a series of 302 French patients with hidradenitis suppurativa, with an analysis of factors associated with disease severity. J Am Acad Dermatol (61): 51-57.

14. Vlassova N, Kuhn D, Okoye GA (2015) Hidradenitis suppurativa disproportionately affects African Americans: a single-center retrospective analysis. Acta Derm Venereol 95: 990-991. [Crossref]

15. Davis SA (2015) Hidradenitis suppurativa management in the united states: an analysis of the national ambulatory medical care survey and market scan medicaid databases. Skin Appendage Disord (2): 65-73.

16. Von der Werth JM, Williams HC (2000) The natural history of hidradenitis suppurativa. J Eur Acad Dermatol Venereol 14(5): 389-392.

17. Schrader AM, Deckers IE, van der Zee HH, Boer J, Prens EP (2014) Hidradenitis suppurativa: a retrospective study of 846 Dutch patients to identify factors associated with disease severity. J Am Acad Dermatol 71: 460-467. [Crossref]

18. Fitzsimmons JS, Guilbert PR, Fitzsimmons EM (1985) Evidence of genetic factors in hidradenitis suppurativa. Br J Dermatol 113: 1-8. [Crossref] 
19. Von Der Werth JM, Williams HC, Raeburn JA (2000) The clinical genetics of hidradenitis suppurativa revisited. Br J Dermatol 142: 947-953. [Crossref]

20. Wang B, Yang W, Wen W, Sun J, Su B, et al. (2010) Gamma-secretase gene mutations in familial acne inversa. Science 330: 1065. [Crossref]

21. Sartorius K, Emtestam L, Jemec GB, Lapins J (2009) Objective scoring of hidradenitis suppurativa reflecting the role of tobacco smoking and obesity. $B r J$ Dermatol 161: 831-839. [Crossref]

22. Vazquez BG (2013) Incidence of hidradenitis suppurativa and associated factors: a population-based study of Olmsted County, Minnesota. J Invest Dermatol 133: 97-103.

23. Bettoli V, Naldi L, Cazzaniga S, Zauli S, et al. (2016) Overweight, diabetes and disease duration influence clinical severity in hidradenitis suppurativa-acne inversa: evidence from the national Italian registry. Br J Dermatol 174: 195-197. [Crossref]

24. Kromann CB (2014) Risk factors, clinical course and long-term prognosis in hidradenitis suppurativa: a cross-sectional study. Br J Dermatol 171(4): 819-824.

25. Dessinioti (2017) A retrospective institutional study of the association of smoking with the severity of hidradenitis suppurativa. $J$ Dermatol Sci in press.

26. Shlyankevich J, Chen AJ, Kim GE, Kimball AB (2014) Hidradenitis suppurativa is a systemic disease with substantial comorbidity burden: a chart-verified casecontrol analysis. J Am Acad Dermatol 71: 1144-1150. [Crossref]

27. Shavit E (2015) Psychiatric comorbidities in 3207 patients with hidradenitis suppurativa. J Eur Acad Dermatol Venereol 29(2): 371-376.

28. Porter ML, Kimball AB (2017) Comorbidities of hidradenitis suppurativa. Semin Cutan Med Surg 36(2): 55-57.

29. Matusiak L (2009) Increased serum tumour necrosis factor-a in hidradenitis suppurativa patients: is there a basis for treatment with anti-tumour necrosis factor-a agents? Acta dermato-venereologica 89(6): 601-603.

30. Gulliver W, Zouboulis CC, Prens E, Jemec GB, Tzellos T (2016) Evidence-based approach to the treatment of hidradenitis suppurativa/acne inversa, based on the European guidelines for hidradenitis suppurativa. Reviews in Endocrine and Metabolic Disorders 17(3): 343-351.

31. Kraft JN, Searles GE (2007) Hidradenitis suppurativa in 64 female patients: retrospective study comparing oral antibiotics and antiandrogen therapy. J Cutan Med Surg 11(4): 125-131.

32. Lee A, Fischer G (2015) A case series of 20 women with hidradenitis suppurativa treated with spironolactone. Australas J Dermatol 56(3): 192-196.

33. Riis PT (2016) The role of androgens and estrogens in hidradenitis suppurativa-a systematic review. Acta dermatovenerol Croat 4(4): 239.

34. Khandalavala BN, Do MV (2016) Finasteride in hidradenitis suppurativa: a "male" therapy for a predominantly "female" disease. J Clin Aesthet Dermatol 9(6): 44.

35. Blok JL, van Hattem S, Jonkman MF, Horváth B (2013) Systemic therapy with immunosuppressive agents and retinoids in hidradenitis suppurativa: a systematic review. Br J Dermatol 168: 243-252. [Crossref]

36. Janse I (2016) Surgical procedures in hidradenitis suppurativa. Dermatol Clin 34(1): 97-109.

37. Blok JL (2015) Surgery under general anaesthesia in severe hidradenitis suppurativa: a study of 363 primary operations in 113 patients. J Eur Acad Dermatol Venereol 29(8): 1590-1597.

38. Danby FW (2015) New and traditional surgical approaches to hidradenitis suppurativa. J Am Acad Dermatol 73(5) S62-5.

39. Hamzavi IH (2015) Laser and light-based treatment options for hidradenitis suppurativa. J Am Acad Dermatol 73(5): S78-81.

40. Lee RA, Eisen DB (2015) Treatment of hidradenitis suppurativa with biologic medications. J Am Acad Dermatol 73: S82-88. [Crossref]

41. Barlev D (2015) Hidradenitis suppurativa: a review with a focus on treatment data. Skin Therapy Lett 20(4): 1-8.

42. Giamarellos-Bourboulis EJ, Pelekanou E, Antonopoulou A, Petropoulou H, Baziaka F, et al. (2008) An open-label phase II study of the safety and efficacy of etanercept for the therapy of hidradenitis suppurativa. Br J Dermatol 158: 567-572. [Crossref]

43. Lee RA (2009) A prospective clinical trial of open-label etanercept for the treatment of hidradenitis suppurativa. J Am Acad Dermatol 60: 565-73.

44. Adams DR, Yankura JA, Fogelberg AC, Anderson BE (2010) Treatment of hidradenitis suppurativa with etanercept injection. Arch Dermatol 146: 501-504. [Crossref]

45. Grant A, Gonzalez T, Montgomery MO, Cardenas V, Kerdel FA (2010) Infliximab therapy for patients with moderate to severe hidradenitis suppurativa: a randomized, double-blind, placebo-controlled crossover trial. J Am Acad Dermatol 62: 205-217. [Crossref]

46. Napolitano M (2017) Hidradenitis suppurativa: from pathogenesis to diagnosis and treatment. Clin Cosmet Investig Dermatol 10: 105.

47. Shuja F, Chan CS, Rosen T (2010) Biologic drugs for the treatment of hidradenitis suppurativa: an evidence-based review. Dermatologic clinics 28(3): 511-24.
48. Kimball AB, Okun MM, Williams DA, Gottlieb AB, Papp KA, et al, (2016) Two Phase 3 Trials of Adalimumab for Hidradenitis Suppurativa. $N$ Engl J Med 375: 422-434. [Crossref]

49. Tzanetakou V, Kanni T, Giatrakou S (2015) Safety and efficacy of anakinra in severe hidradenitis suppurativa: a randomized clinical trial. JAMA Dermatol 1-9. [epub]

50. Matusiak L, Szczech J, Bieniek A, Nowicka-Suszko D, Szepietowski JC (2017) Increased interleukin (IL)-17 serum levels in patients with hidradenitis suppurativa: Implications for treatment with anti-IL-17 agents. Journal of the American Academy of Dermatology 76(4): 670-675.

51. Thorlacius L, Theut Riis P, Jemec GB (2018) Severe hidradenitis suppurativa responding to treatment with secukinumab: a case report. British Journal of Dermatology

52. Gulliver WP, Jemec GB, Baker KA (2012) Experience with ustekinumab for the treatment of moderate to severe hidradenitis suppurativa. Journal of the European Academy of Dermatology and Venereology 26(7): 911-914.

53. Blok JL, Li K, Brodmerkel C, Horvátovich P, Jonkman MF, Horváth B (2016) Ustekinumab in hidradenitis suppurativa: clinical results and a search for potential biomarkers in serum. British Journal of Dermatology 174(4): 839-846.

54. Alikhan A, Lynch PJ, Eisen DB (2009) Hidradenitis suppurativa: a comprehensive review. J Am Acad Dermatol 60(4): 539-561.

55. Zouboulis CC (2015) European S1 guideline for the treatment of hidradenitis suppurativa/acne inversa. J Eur Acad Dermatol Venereol 29(4): 619-644.

56. Wang SC (2015) Hidradenitis Suppurativa: A Frequently Missed Diagnosis, Part 2: Treatment Options. Adv Skin Wound Care 28(8): 372-380.

57. Riis PT (2016) Intralesional triamcinolone for flares of hidradenitis suppurativa (HS): A case series. J Am Acad Dermatol 75(6): 1151-1155.

58. Levine RM, Rasmussen JE (1983) Intralesional corticosteroids in the treatment of nodulocystic acne. Arch Dermatol 119: 480-481. [Crossref]

59. Jansen T (2000) Disfiguring draining sinus tracts in a female acne patient. Pediatr Dermatol 17(2): 123-125.

60. Hsiao JL, Antaya RJ, Berger T, Maurer T, Shinkai K, et al. (2010) Hidradenitis suppurativa and concomitant pyoderma gangrenosum: a case series and literature review. Arch Dermatol 146: 1265-1270. [Crossref]

\section{Citation:}

Erika L. Crowley, Claudia J. Posso-De Los Rios, Melinda J. Gooderham (2018)

Retrospective Study of 133 Canadian Hidradenitis Suppurativa Patients and Literature Review. Internal Med Res Open J Volume 3(2): 1-6 